\title{
Erratum
}

Toufik Khairat ${ }^{1}$ Mohammed Salah' / Khadija Marakchi' / Najia Komiha'

\section{Erratum to: Theoretical Study of the Electronic States of Newly Detected Dications. Case of $\mathrm{MgS}^{2+}$ AND SiN $^{2+}$}

${ }^{1}$ Laboratoire LS3ME, Faculty of Sciences Rabat, University Mohammed V, Rabat, Morocco, E-mail: komiha@fsr.ac.ma

DOI: $10.1515 /$ psr-2016-9101

Erratum to: Toufik Khairat, Mohammed Salah, Khadija Marakchi, Najia Komiha. "Theoretical study of the electronic states of newly detected dications. Case of $\mathrm{MgS}^{2+} \mathrm{AND} \mathrm{SiN}^{2+\prime \prime}$, in: PSR: issue 9, volume 2 (2017), DOI https://doi.org/10.1515/psr-2016-5101.

As the original DOI (https://doi.org/10.1515/psr-2016-0101) was a duplicate, a new DOI has now been assigned. The new DOI is https://doi.org/10.1515/psr-2016-5101. 